\title{
Transcallosal approach for tumors of the lateral and third ventricles
}

\author{
Hahnah Kasowski, M.D., AND JosePh M. Piepmeier, M.D. \\ Department of Neurosurgery, Yale University School of Medicine, New Haven, Connecticut
}

\begin{abstract}
Tumors that arise within the ventricles present a unique surgical challenge. Because of their deep location, relatively large size, and their association with hydrocephalus, surgical planning requires a careful assessment of the optimal method to access the lesion and to provide adequate exposure for tumor resection. The transcallosal approach to the lateral ventricles often is the best procedure by which to achieve these goals.

Partial sectioning of the corpus callosum does not cause significant neurological deficits; however, if the surgery induces additional brain injury, the neurological deficits can be more severe in the presence of a callosotomy. Knowledge of the techniques of transcallosal surgery and careful preoperative planning can reduce the risk of permanent neurological impairment; these range from protection of the cortical veins that drain into the superior sagittal sinus to brain relaxation and ventricular drainage, as well as proper identification of anatomical landmarks within the ventricle. The transcallosal approach can offer a relatively easy access to the lateral and third ventricles, and with proper planning it can reduce the morbidity associated with resection of lesions within these compartments.
\end{abstract}

\author{
KEY WORDS • intraventricular tumor • transcallosal approach • hydrocephalus • \\ lateral ventricle
}

Planning for any neurosurgical operation should begin with an assessment of what is the best procedure based on the anticipated pathological entity. The surgeon must plan for a procedure that can be performed with minimal or acceptable morbidity. Finally, surgical intervention must offer a better outcome than that associated with the known natural history of nonsurgical treatment.

Because lateral and third ventricular tumors are relatively rare, there are few neurosurgeons with extensive experience in their management. The majority, however, are benign or low-grade tumors, and surgery is often the best option for treatment. ${ }^{11,12}$ The selection of an appropriate operative approach to the ventricles is a fundamental aspect of that process.

Some of the initial reports on transcallosal surgery were published in the mid-1970s, ${ }^{6,713}$ and since that time others have reported their experience. ${ }^{1,3,8,10-12,18}$ In general, partial disconnection of the hemispheres does not result in a significant neurological deficit; however, serious consequences arise if patient selection criteria are inadequate or the technique is not carefully planned and executed. ${ }^{4,6,7,9}$, $14,15,17,19$ In this report we focus on patient selection criteria, operative technique, and postoperative care of patients in

\footnotetext{
Abbreviation used in this paper: $\mathrm{MR}=$ magnetic resonance.
}

whom the transcallosal approach has been used to resect tumors in the lateral and third ventricles. Although this procedure can help to achieve appropriate surgical goals, inexperience and lack of attention to details can result in serious and permanent neurological impairment.

\section{TRANSCALLOSAL APPROACH TO LATERAL AND THIRD VENTRICLES}

\section{Patient Selection}

Although it provides limited access to tumors in the posterior trigone, temporal horn or superior frontal horn, the transcallosal approach to the lateral and third ventricles can provide maximal exposure to tumors arising in these regions (Fig. 1 left). Patients harboring tumors arising in these locations are best managed by transcortical approaches (Fig. 1 right). Each of these surgical options has its own set of potential complications. ${ }^{12,16}$

One of the contraindications for transcallosal surgery is crossed dominance, a condition in which the hemisphere controlling the dominant hand is contralateral to the hemisphere mediating language and speech. ${ }^{4,5,9,14,15}$ Crossed dominance can occur when there is evidence of extracallosal dysfunction, particularly following cerebral injury (due to surgery, trauma, or infection) during childhood 


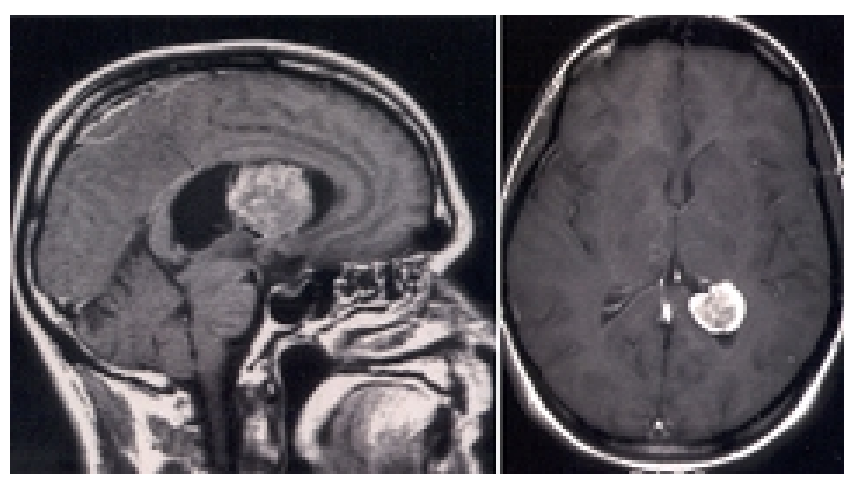

Fig. 1. Left: Sagittal gadolinium-enhanced MR image revealing a neurocytoma arising in the region of the septum pellucidum. This tumor is in an ideal location for a transcallosal approach. Right: Axial gadolinium-enhanced MR image demonstrating a meningioma arising in the posterior trigone region of the lateral ventricle. This lesion is difficult to reach via a transcallosal approach and is best treated by a transcortical procedure.

and resulting in relocation of function. These patients may be at risk for writing- and speech-related deficits following callosal sectioning. Transcallosal surgery may also be contraindicated if the splenium of the corpus callosum is sectioned in the presence of a homonymous hemianopia in the dominant hemisphere, causing alexia.

Most patients with intraventricular tumors reach medical attention because of the signs and symptoms of hydrocephalus that have caused cognitive disabilities such as memory, perception, and fine motor skill deficits..$^{10-12}$ Because the risk of causing memory deficits is also a concern should injury to the fornices occur, $, 6,7,15,17,19$ a neuropsychological evaluation for cognitive deficits can be an important part of the preoperative assessment.

Significant neurological impairment may occur when the hemispheres are damaged operatively. ${ }^{11,12}$ This most common deficit is associated with excessive retraction of a hemisphere in the presence of hydrocephalus, loss of a dominant cortical vein, venous infarction, or inaccurate identification of anatomy and excessive manipulation of compromised white matter tracts within the ventricle.

\section{Operative Approaches}

Because the interhemispheric approach provides equal access to both lateral ventricles, the side of the craniotomy is primarily dependent on the presence of midline draining cortical veins. If venous drainage is symmetrical, the nondominant hemisphere is the optimal choice; however, preservation of venous drainage overrides other considerations for placement of the craniotomy. Magnetic resonance venography can usually provide adequate visualization of the venous drainage for preoperative planning. Exposure of the interhemispheric region requires extending the craniotomy to the midline over the superior sagittal sinus. To access the interhemispheric fissure, removal of a bone flap up to the edge of the superior sagittal sinus can be followed by careful separation of the sinus from the inner table of the skull and then removal of the bone over the sinus.

The dural opening typically begins laterally and is carefully extended toward the midline. It is not uncommon to find that dominant frontal veins enter the dura lateral to the superior sagittal sinus margin. When this is encountered, the dural opening can be redirected; this is performed to leave a dural roof by incising on both sides of the vein. The goal of creating the dural opening is to bring the operative exposure to the edge of the sinus. Retraction of the dura over the sinus should be performed so that blood flow is not impaired (Fig. 2).

Brain relaxation is critically important to minimize retraction-induced injury and should occur prior to interhemispheric dissection. To this end, hyperventilation, osmotic diuresis, and ventricular drainage systems are used.

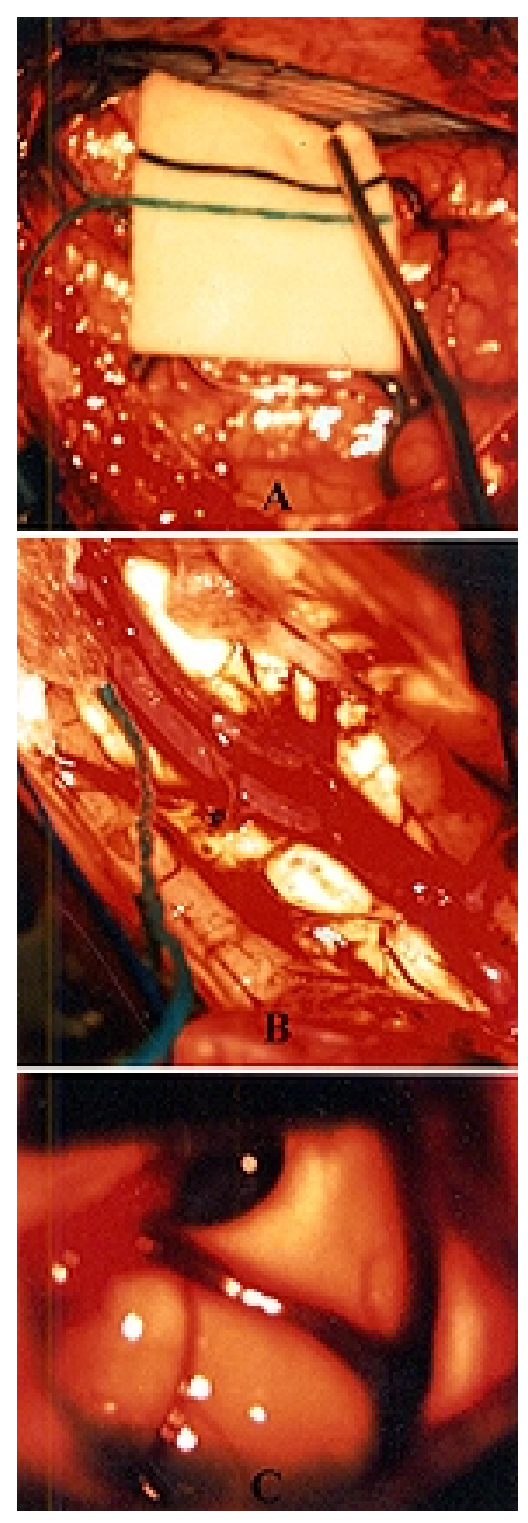

Fig. 2. Intraoperative photographs. A: Initial surgical access to the interhemispheric fissure. Brain relaxation and preservation of midline veins permit safe access to the corpus callosum. B: The anterior cerebral arteries overlie the corpus callosum. These arteries can be easily separated to allow access to the corpus callosum. C: The lateral ventricle viewed through the operating microscope. The choroid plexus is seen entering the foramen of Monro. 
The ventricular drain is often placed following dural opening ipsilateral to the craniotomy; however, when large tumors preclude ipsilateral placement, use of the contralateral ventricle remains an option.

The arachnoid granulations found along the medial hemisphere into the sagittal sinus are opened with sharp dissection, and interhemispheric dissection is begun. At this point the operating microscope is used. The dissection requires identification of both anterior cerebral arteries and opening of the callosum between them. The corpus callosum is identified by its relative hypovascularity and pale white color. The length and placement of the callosal sectioning is determined by the location and size of the tumor.

Once the ventricle is opened, it is important first to establish normal landmarks. Identification of the choroid plexus, septal vein, and thalamostriate vein all will lead to the foramen of Monro. If the callosal sectioning has led to the contralateral ventricle, the septum pellucidum can be opened to access the ipsilateral side. Should this occur, it is imperative to preserve the fornices at the base of the septum. Tumor resection is facilitated by maintaining the dissection plane between the ependyma and lesion. If the foramen of Monro is patent, placement of a barrier will prevent blood from pooling into the third ventricle, avoiding potential ventricular obstruction.

There are several options for accessing the third ventricle via the transcallosal approach. The foramen of Monro provides access to the anterior third ventricle; however, lesions in the middle and posterior third ventricle require an alternative exposure. An expanded foramen does not routinely need to be enlarged by sacrifice of a fornix. If a lesion located within the third ventricle can be decompressed and delivered into the foramen, then this is the most appropriate use of this approach.

A common approach to the middle and posterior third ventricle is through the choroidal fissure into the velum interpositum (Fig. 3). This space is defined laterally by the insertion of the choroid plexus, superiorly by the fornix, and inferiorly by the dorsomedial thalamus. The choroid plexus can be removed along its insertion in the choroidal fissure. The fornix is then gently elevated along with the internal cerebral vein, which opens the velum interpositum. The tumor is decompressed into the area of exposure and mobilized away from the ependymal surface of the ventricle.

The final transcallosal approach to the third ventricle is through interforniceal dissection. Because the anatomical definition of this space may be difficult to identify and because bilateral forniceal injury can cause severe memory deficits, this approach is often reserved for cases in which there is either a cavum septum pellucidum or significant mass effect that has anatomically separated the fornices.

\section{Tumor Removal}

It is not uncommon for lesions within the ventricles to grow and expand slowly, and these tumors can become quite large before they are identified. Consequently, the surgery must be planned to reach the lesion and to decompress it so that it can be removed via a relatively small opening. Tumors arising in the choroid plexus such as

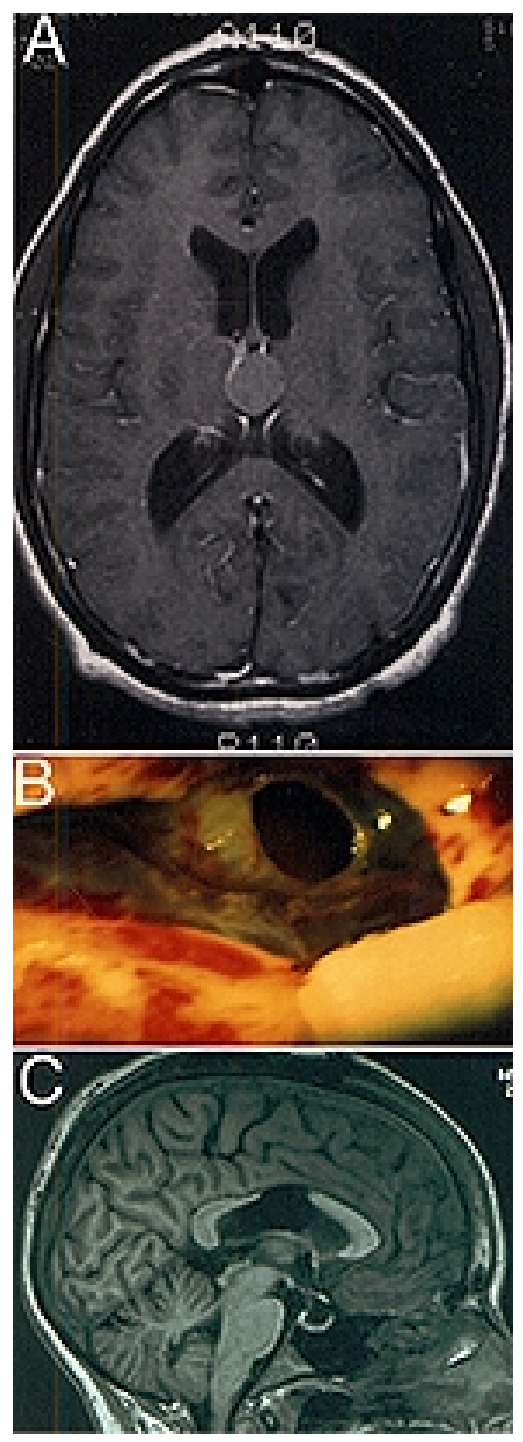

Fig. 3. A: Axial gadolinium-enhanced MR image revealing a colloid cyst within the third ventricle. B: Intraoperative photograph demonstrating the opened cyst accessed via the choroidal fissure. The choroid plexus has been removed, and the fornix is seen over the top of the cyst. A white probe is pointing to the dorsomedial thalamus. C: Postoperative nonenhanced MR image demonstrating the opening in the corpus callosum for removal of the colloid cyst.

papillomas and meningiomas receive their blood supply from the choroidal vessels (Fig. 4). Early identification and transection of these vessels will reduce bleeding associated with piecemeal tumor resection. Lesions that arise from the ependymal surface and septum pellucidum (for example, gliomas and neurocytomas) will receive blood supply from the small vessels of the ventricular walls. These small vessels create less intraoperative blood loss, but because they are frequently numerous and small, meticulous dissection with microscopic illumination and magnification will be mandatory.

Intraventricular surgery requires the surgeon to work in a small space and to manipulate a tumor several centimeters from the brain surface. To prevent misidentification of 


\section{H. Kasowski and J. M. Piepmeier}

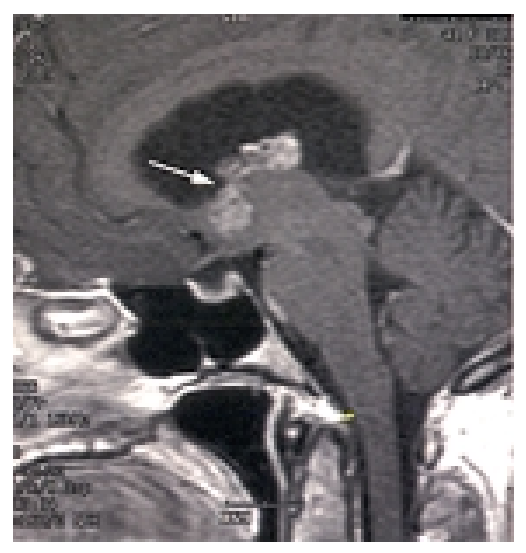

Fig. 4. Sagittal gadolinium-enhanced MR image revealing a meningioma (arrow) arising in the lateral ventricle and extending into the third ventricle.

normal anatomy, it is helpful to orient the dissection according to reliable anatomical landmarks. The course of the choriod plexus and the thalamostriate vein to the foramen of Monro, where it is joined by the septal vein and the vein of the caudate to form the internal cerebral vein, can be used to guide the surgeon for localization of the fornix, thalamus, and septum pellucidum. When increased magnification restricts the field of view through the operating microscope, it is frequently helpful to become reoriented to the normal anatomy to prevent wandering into the thalamus, hypothalamus, and fornix. Whenever possible, the plane between the tumor and ependymal surface should be maintained.

The use of neuroendoscopy has become common for surgery within the lateral ventricles. ${ }^{2}$ Endoscopy can provide visualization of and access to both the body of the lateral ventricles as well as the third ventricle with minimal manipulation of the brain (Fig. 5). Cystic lesions can be aspirated and biopsy samples obtained using existing technology. Endoscopy also can be useful as a surgical adjuvant during microsurgical excision of large intraventricular tumors.

Following tumor resection, it is important to ensure complete hemostasis. To prevent delayed ventricular obstruction, care is taken to irrigate blood that may have pooled in the lateral and third ventricles. The ventricles are filled with warm saline at the end of the surgery to remove air that may have become trapped. A ventricular catheter is left in the lateral ventricle for approximately 48 hours postoperatively to monitor intracranial pressure and to demonstrate that the ventricular system is patent. It is routine to obtain a computerized tomography scan on the 1 st postoperative day to evaluate the extent of the tumor resection and to check for obstruction. The catheter can be removed at the bedside prior to mobilization of the patient.

\section{CONCLUSIONS}

The transcallosal approach to the lateral and third ventricle lesions offers an important surgical option. There are several advantages to this approach over the transcortical route. With appropriate brain relaxation and minimal

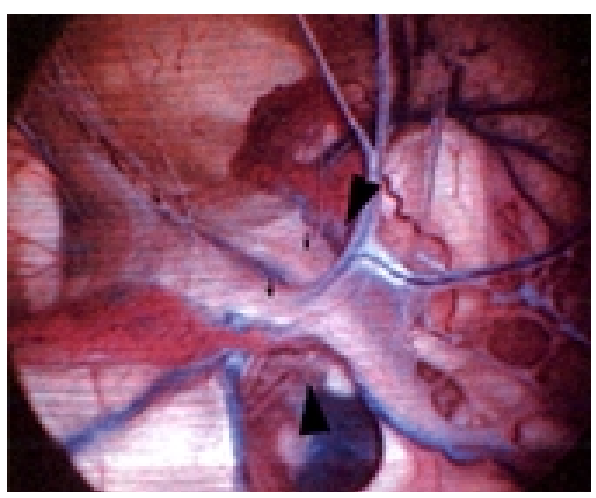

Fig. 5. Intraoperative endoscopic photograph of the lateral ventricles. This patient has hydrocephalus associated with agenesis of the septum pellucidum. Large arrowheads indicate the choroid plexus from the right and left lateral ventricles entering the foramen of Monro. Small arrows point to the fornices.

retraction, the surgeon can reduce the risk of brain injury. Care to preserve medial draining veins prevents venous infarction. Partial sectioning of the callosum leads to minimal long-term consequences unless additional brain injury is induced. Because this approach is not commonly used in the average neurosurgical practice, it is important to review the anatomy and to pay careful attention to the techniques that optimize a favorable outcome.

Transcallosal surgery carries a reduced risk of postoperative seizures, porencephalic cyst formation, and subdural hygroma formation compared with transcortical surgery. ${ }^{11,12,16}$ Limited exposure of the posterior lateral ventricle and the apex of the frontal horn, however, reduces the utility of the transcallosal approach for lesions arising in these regions.

Because the primary neurological signs and symptoms associated with intraventricular tumors are caused by hydrocephalus, ${ }^{11,12}$ it is important to ensure that the resection of the lesion has opened the ventricular pathways. Prolonged ventricular dilation induced by a long-standing lesion may not be reversible; these patients should be monitored as it is possibile; they may have a condition similar to normal-pressure hydrocephalus. The failure to achieve anticipated recovery of cognitive abilities after tumor resection is an indication that additional testing should be undertaken. In selected patients in whom to improvement does not occur, placement of a ventricular shunt may be required. ${ }^{11,12}$

\section{References}

1. Ehni G: Interhemispheric and percallosal (transcallosal) approach to the cingulate gyri, Intraventricular shunt tubes, and certain deeply placed brain lesions. Neurosurgery 14: 99-110,1984

2. Fries G, Perneczky A: Endoscopic-assisted brain surgery: Part 2-analysis of 380 procedures. Neurosurgery 42:226-232, 1998

3. Geffen G, Walsh A, Simpson D, et al: Comparison of the effects of transcortical and transcallosal removal of intraventricular tumours. Brain 103:773-788, 1980

4. Geschwind N: Disconnexion syndromes in animals and man. Brain 88:237-294, 1965 


\section{Transcollosal approach for lateral and third ventricle tumors}

5. Heilman KM, Watson RT, Valenstein E, et al: Localization of lesions in neglect, in Heilman K, Valenstein E (eds): Clinical Neuropsychology. New York: Oxford University Press, 1985, pp 243-293

6. Jeeves MA, Simpson DA, Geffen G: Functional consequences of the transcallosal removal of intraventricular tumours. J Neurol Neurosurg Psychiatry 42:134-142, 1979

7. Ledoux JE, Risse GL, Springer SP, et al: Cognition and commissurotomy. Brain 100:87-104, 1977

8. Lejeune J, Le Gars D, Haddad E: [Tumors of the third ventricle: review of 262 cases.] Neurichirurgie 46:211-238, 2000 (Fr)

9. Levin HS, Rose JE: Alexia without agraphia in a musician after transcallosal removal of a left intraventricular meningioma. Neurosurgery 4:168-174, 1979

10. Piepmeier JM: Tumors and approaches to the lateral ventricles. Introduction and overview. J Neurooncol 30:267-274, 1996

11. Piepmeier JM, Sass KJ: Surgical management of lateral ventricular tumors, in Paoletti P, Takakura K, Walker MD, et al (eds): Neuro-Oncology. Boston: Kluwer Academic Publishers, 1991, pp 333-335

12. Piepmeier JM, Spencer DD, Sass KJ, et al: Lateral ventricular masses, in Apuzzo MLJ (ed): Brain Surgery: Complication Avoidance and Management. New York: Churchill Livingstone, 1993, Vol 1, pp 581-600

13. Shucart WA, Stein BM: Transcallosal approach to the anterior ventricular system. Neurosurgery 3:339-343, 1978

14. Sass K, Westerveld M, Novelly R, et al: Intracarotid amytal procedure findings predict post-callosotomy motor impairments in right hemisphere speech dominant epileptic patients. Epilepsia 30:711-712, 1989

15. Sass K, Novelly R, Spencer D, et al: Mnestic and attention impairments following corpus callosotomy section for epilepsy. J Epilepsy 1:61-66, 1988

16. Tanaka Y, Sugita K, Kobayashi S, et al: Subdural fluid collections following transcortical approach to intra- or paraventricular tumours. Acta Neurochir 99:20-25, 1989

17. Westerveld M, Sass K, Spencer S, et al: Neuropsychological function following corpus callosotomy for epilepsy, in Devinsky O, Theodore W (eds): Epilepsy and Behavior. New York: Alan Liss, 1991, pp 203-212

18. Winkler PA, Ilmberger J, Krishnan KG, et al: Transcallosal interforniceal-transforaminal approach for removing lesions occupying the third ventricular space: clinical and neuropsychological results. Neurosurgery 46:879-890, 2000

19. Zaidel D, Sperry RW: Memory impairment after commissurotomy in man. Brain 97:263-272, 1974

Manuscript received April 18, 2001.

Accepted in final form May 15, 2001.

Address reprint requests to: Joseph M. Piepmeier, M.D., Department of Neurosurgery, Yale University School of Medicine, 333 Cedar Street, New Haven, Connecticut 06520.email: joseph. piepmeier@yale.edu. 